\title{
Three-dimensional structure of Mycoplasma pneumoniae's attachment organelle and a model for its role in gliding motility
}

\author{
Gregory P. Henderson and Grant J. Jensen* \\ Division of Biology, California Institute of Technology, \\ Pasadena, CA, USA.
}

\section{Summary}

While most motile bacteria propel themselves with flagella, other mechanisms have been described including retraction of surface-attached pili, secretion of polysaccharides, or movement of motors along surface protein tracks. These have been referred to collectively as forms of 'gliding' motility. Despite being simultaneously one of the smallest and simplest of all known cells, Mycoplasma pneumoniae builds a surprisingly large and complex cell extension known as the attachment organelle that enables it to glide. Here, three-dimensional images of the attachment organelle were produced with unprecedented clarity and authenticity using state-of-the-art electron cryotomography. The attachment organelle was seen to contain a multisubunit, jointed, dynamic motor much larger than a flagellar basal body and comparable in complexity. A new model for its function is proposed wherein inchworm-like conformational changes of its electron-dense core are leveraged against a cytoplasmic anchor and transmitted to the surface through layered adhesion proteins.

\section{Introduction}

The mycoplasmas are simultaneously the smallest and simplest of known cells. Volumes can be $\sim 25$ times smaller than Escherichia coli (Biberfeld and Biberfeld, 1970), and their genomes can be limited to only several hundred genes (Fraser et al., 1995; Himmelreich et al., 1996). Despite the pressures that drove them to such minimization, amazingly, some construct a complex structure at their tips called the attachment organelle whose predicted mass is greater than that of a vertebrate nuclear pore complex! In Mycoplasma pneumoniae this attachment organelle is essential for cytadherence (Baseman

Accepted 7 February, 2006. *For correspondence. E-mail jensen@ caltech.edu; Tel. (+1) 626395 8827. Fax (+1) 6263955730.

(C) 2006 The Authors

Journal compilation @ 2006 Blackwell Publishing Ltd et al., 1982; Morrison-Plummer et al., 1986) and motility (Balish et al., 2003; Hasselbring et al., 2005; Seto et al., 2005a), but the mechanisms are unknown.

Various types of motility have been described in prokaryotes. While the most common type is flagellar, a number of non-flagellar, so-called 'gliding' forms of movement also exist. The 'twitching' motility of Pseudomonas aeruginosa and the 'social' motility of Myxococcus xanthus are effected by cells extending and retracting surface-attached type IV pili (Mattick, 2002). Filamentous cyanobacteria and the 'adventurous' motility of $M$. xanthus rely on the secretion of polysaccharide slime (McBride, 2001). Flavobacterium johnsoniae is thought to move by a treadmilling mechanism involving surface protein that move along tracks on the cell surface (McBride, 2001). Even among the motile mycoplasmas, various forms of motility appear to exist. Mycoplasma mobile relies on three large surface proteins (Uenoyama et al., 2004; Seto et al., 2005b; Uenoyama and Miyata, 2005), but these proteins lack clear homologues in other motile mycoplasma species such as Mycoplasma genitalium and M. pneumoniae (Miyata, 2005). Instead, these organisms' motility appears to depend on the attachment organelle, which therefore probably underlies an entirely unique and interesting form of gliding motility.

Mycoplasma pneumoniae causes bronchitis and atypical pneumoniae in humans by binding to the respiratory epithelium using surface proteins localized by the attachment organelle. Adhesion P1 (169 kDa) and accessory protein P30 $(30 \mathrm{kDa})$ are necessary for this adhesion (Morrison-Plummer et al., 1986) and for cell motility (Hasselbring et al., 2005; Seto et al., 2005a). Specifically P30 has been proposed to serve as a link between the force generation mechanism and the surface adhesion proteins (Hasselbring et al., 2005). Other surface proteins include protein $B(90 \mathrm{kDa})$ and protein $\mathrm{C}(40 \mathrm{kDa})$, which help to localize P1 (Baseman et al., 1982). Proteins P65 and the HMWs1-3 are associated with the organelle, but their spatial arrangements and functions are unknown (Krause and Balish, 2004). A massive protein assembly over $220 \mathrm{~nm}$ long and $50 \mathrm{~nm}$ thick known as the 'electron-dense core' occupies the centre of the attachment organelle (Biberfeld and Biberfeld, 1970; Wilson and Collier, 1976). Current characterizations of the core describe 
it as two uniform, striated rods separated by a thin gap (Meng and Pfister, 1980; Hegermann et al., 2002). A distal enlargement of the core has been referred to as the terminal button. The proximal end of the electron-dense core has been proposed to connect to a so-called 'wheellike complex' thought to be composed of two rings of proteins that connect to radial spokes connecting to the membrane (Hegermann et al., 2002). In both the attachment organelle and the cytoplasm, $5 \mathrm{~nm}$ fibres have been reported (Meng and Pfister, 1980; Gobel et al., 1981).

Studying the macromolecular structures in M. pneumoniae has proven difficult. Methods for genetic manipulation are still developing. Light microscopy is limited to resolving the relative positions of labelled proteins along the length of the cell. While electron microscopy has the resolving power to visualize large protein complexes, traditional plastic-embedding methods have obscured important details. Electron cryotomography (ECT) is an emerging technique that can produce threedimensional images of intact cells no thicker than about half a micron, in a life-like, 'frozen-hydrated' state (Lucic et al., 2005). Here, we have used ECT to image the attachment organelle of M.pneumoniae with unprecedented clarity and authenticity. It was seen to be composed of at least 11 distinct protein structures surrounded by a curious electron-lucent area and a membrane studded with organized surface proteins. Based on these results, we propose a model for its role in a new form of gliding motility.

\section{Results \\ Location of the electron-dense core within a cell}

Twelve imaged M. pneumoniae cells were reconstructed (Fig. 1) containing a total of 19 attachment organelles (Fig. 2). The attachment organelles were marked by colocalization of an electron-dense core beneath the membrane, an electron-lucent area surrounding the core, and packed surface proteins. As expected from previous work, we found cells where the electron-dense core protruded out away from the body of the cell within a membranous finger-like extension (Fig. 2A-I). With our population of cells, however, nearly half of the cells had the electrondense core fully internalized into the cell's body, lying next to the cell membrane with only the head of the terminal button maintaining contact with the membrane (Fig. $2 \mathrm{~J}-$ N).

\section{Membrane proteins}

Surface proteins were found to form tightly packed rows $\sim 5.5 \mathrm{~nm}$ thick on the extracellular surface of the attachment organelle (component ' $A$ ' of the schematic in Fig. 2 and highlighted in Fig. 3). These rows localized over the terminal button and extended down the attachment organelle over the electron-lucent areas. Another layer of proteins was seen immediately adjacent to the membrane inside the cell (component ' $\mathrm{B}$ ', pointed to specifically in Fig. 4A).

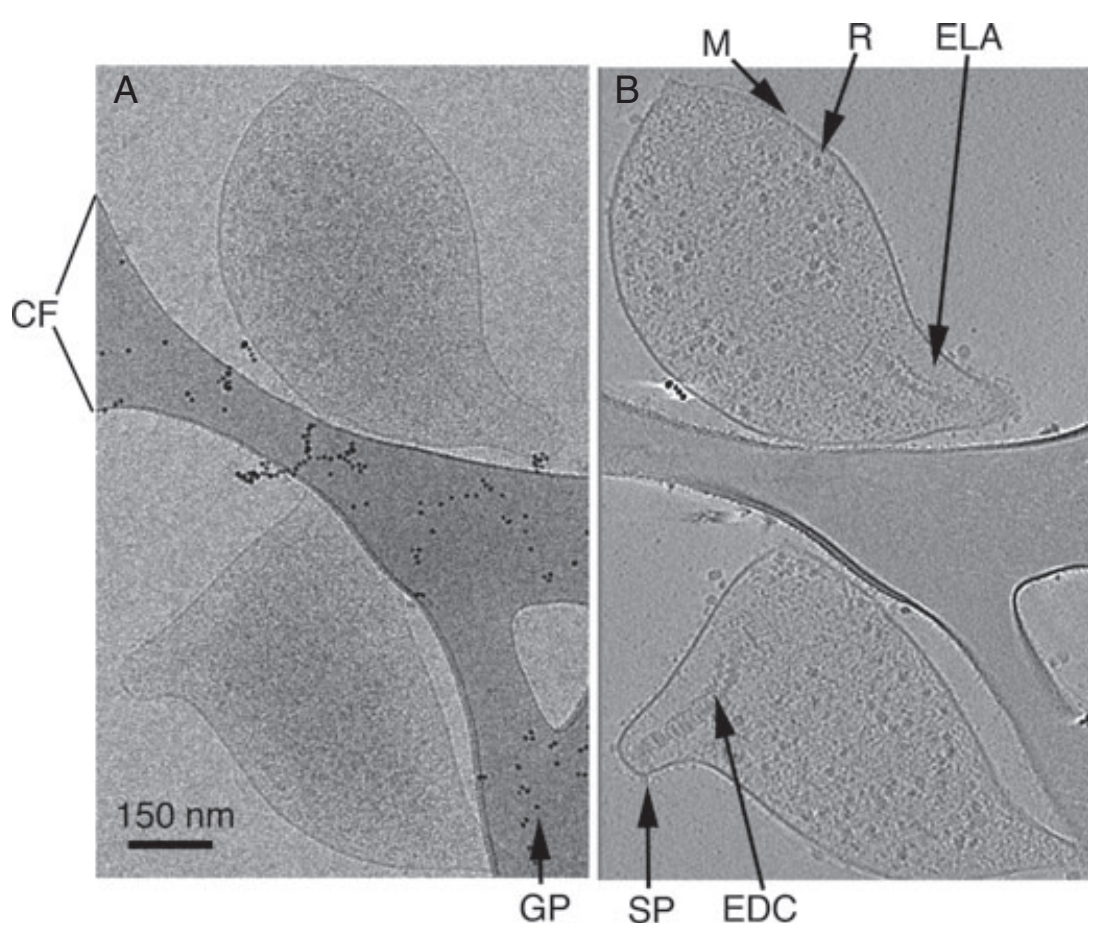

Fig. 1. Electron micrograph and tomographic reconstruction of a dividing Mycoplasma pneumoniae cell.

A. An example untilted projection image from the tilt-series of a single frozen-hydrated cell. The cell appears about to divide and is stretched over the carbon film of the grid. B. A $15 \mathrm{~nm}$ central slice through the threedimensional reconstruction of the same cell perpendicular to the beam. Individual macromolecular complexes are visible, including the cell's two electron-dense cores. (These cores appear again in Fig. 2F and E).

CF, carbon film; EDC, electron-dense core; ELA, electron-lucent area; GP, gold particle; $\mathrm{M}$, membrane; $\mathrm{R}$, ribosome-like particle; SP, surface proteins. 

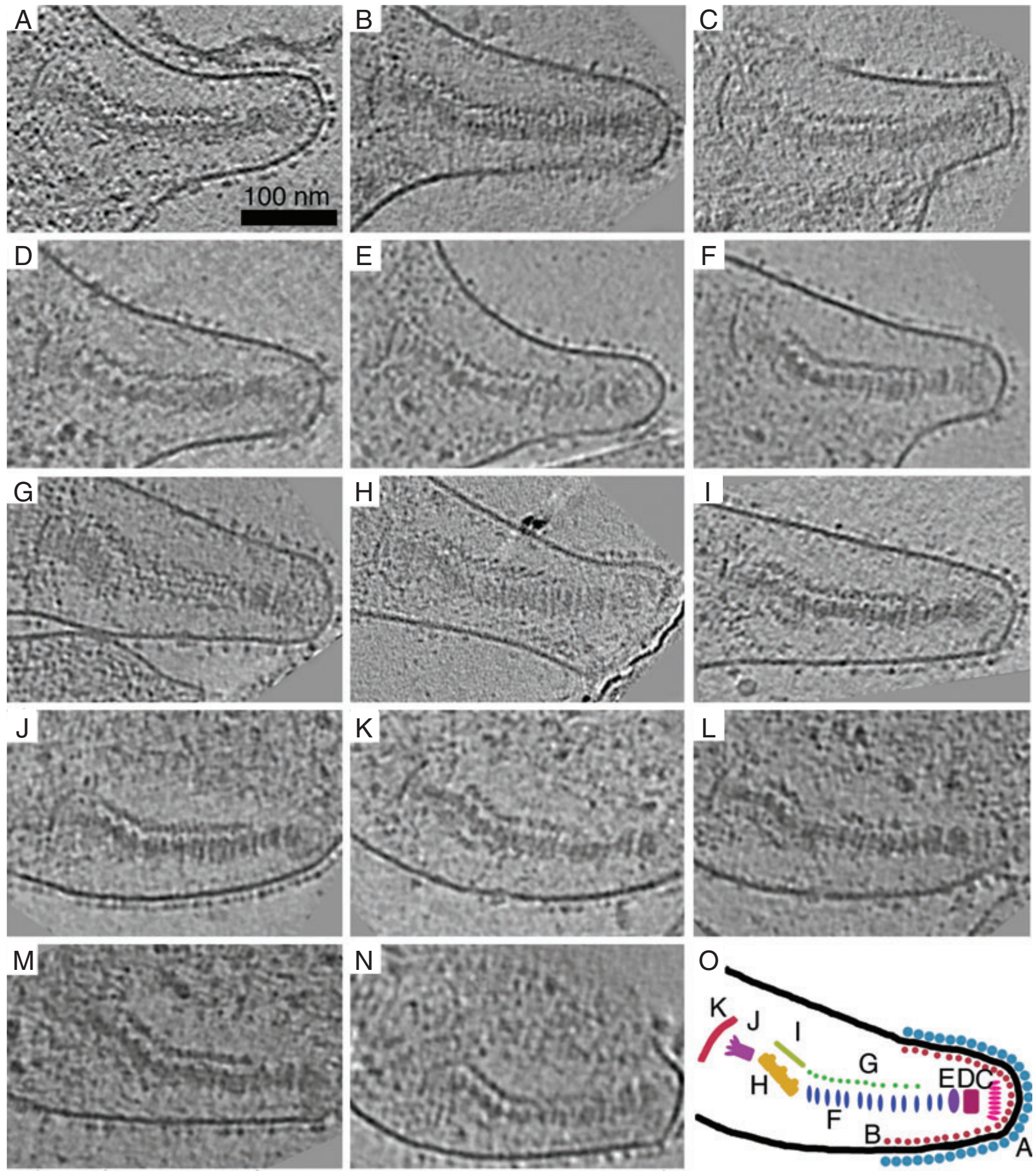

Fig. 2. Montage of attachment organelles and schematic. Each image represents a 10.7- to 22.4-nm-thick slice through a tomogram oriented to expose the thick and thin rods of the electron-dense core. (Five additional cores were considered in the analysis but are not shown here.) A-I. Cores that protruded from the body of the cell within a membrane-enclosed, finger-like extension. J-N. Internalized cores.

O. Schematic of the attachment organelle with components labelled for subsequent reference. 


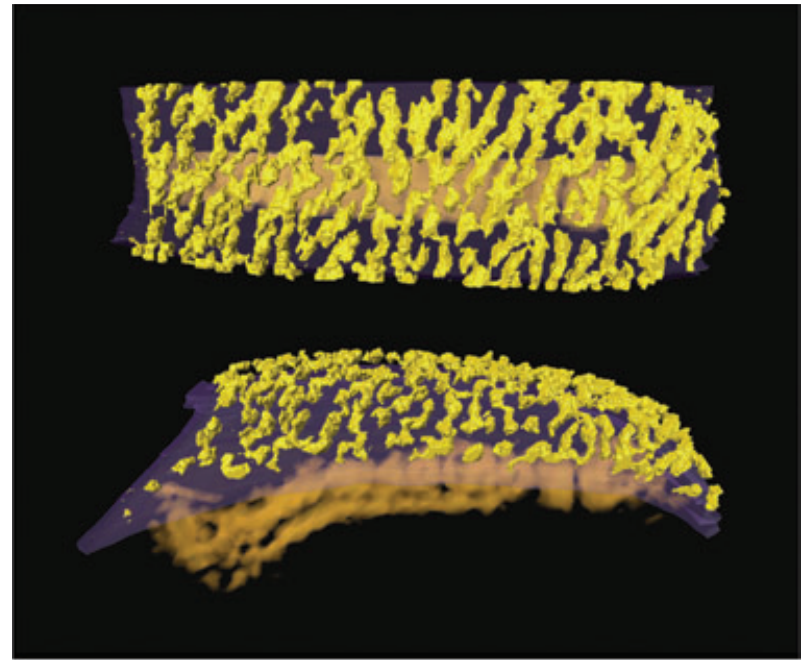

Fig. 3. Extracellular surface proteins. Two views of the attachment organelle pictured in Fig. $2 \mathrm{~J}$ are shown, tilted with respect to each other. The extracellular surface proteins were automatically segmented with a simple density threshold and surface-rendered in yellow. The membrane below was manually segmented and surfacerendered in purple. The electron-dense core was volume-rendered in orange. Because the views are three-dimensional with perspective, no scale bar is included.

\section{Electron-dense core}

The distal end of the electron-dense core has been called the terminal button. Here, the terminal button was seen to be composed of at least three parts. Most distally, there was an arched patch of discrete globular proteins (component ' $C$ ', pointed to specifically in Fig. 4A) that appeared to contact the inner layer of peripheral membrane proteins. More proximally the terminal button contained two nodules (components ' $D$ ' and ' $E$ ') with a gap between them perpendicular to the axis of the core (Fig. 4B). The two nodules did not appear to be completely separated, but instead were probably connected at points around their edges. The more proximal nodule (component 'E') made contact with one of the rods of the electron-dense core. Two parallel rods of different thicknesses and lengths made up the majority of the core, and these were both bent $\sim 150^{\circ}$ just proximal to their midpoint (Fig. 5A). The outer rod (components ' $F$ ' and ' $H$ ') was thicker and varied in thickness from 13 to $31 \mathrm{~nm}$. It was also longer, and was the one that eventually made contact with the terminal button. The thinner rod (components ' $G$ ' and 'I') appeared along the inner curvature and was $\sim 8 \mathrm{~nm}$ in width. Between these two rods was a gap of $\sim 7 \mathrm{~nm}$ (Fig. 5B). The morphology of both the thick and thin rods changed after the bend. After careful study of the structure in three dimensions, it was seen that distal to the bend both rods (components ' $F$ ' and ' $G$ ') were discretely segmented like a vertebral column with gaps perpendicular to the axis of the core; proximal to the bend (components 'I' and ' $\mathrm{H}$ '), the rods were continuous (Fig. 5C). There were about 12 segments plus one or two additional segments in the thick rod that formed the connection with the terminal button. While the core was clearly made of two rods with multiple segments each, extensive contacts were also apparent which presumably explain how the core maintains its integrity even through partial purification. To investigate these contacts a computational 'fill' tool was used to identify all the voxels with a density above a certain threshold that touched one another in the region of the core (Fig. 5D). When these voxels were rendered with a single surface (Fig. 5E), numerous connections between individual segments and the two rods were seen. In total, the cores (components 'C'-'l') measured $\sim 255 \mathrm{~nm}$ in length, and their volumes corresponded to a molecular weight of > 200 MDa.

\section{Bowl complex}

A shallow bowl-like complex was observed proximal to the core in some, but not all of the attachment organelles (component ' $\mathrm{K}$ ', highlighted in Fig. 6). This bowl complex capped the core, but the distance and angle between the two varied (see also Fig. 9 and its discussion below). Contrary to an earlier report, which described it as 'wheellike' (Hegermann et al., 2002), no spokes radiating perpendicular to the core axis were observed. A new density (component ' $J$ '), however, was seen connecting the bowl complex to the electron-dense core.

\section{Electron-lucent area}

Every core was surrounded by a curious electron-lucent area, irrespective of whether the attachment organelle protruded from the cell body or was internalized. Even though no barrier such as a protein wall or membrane was
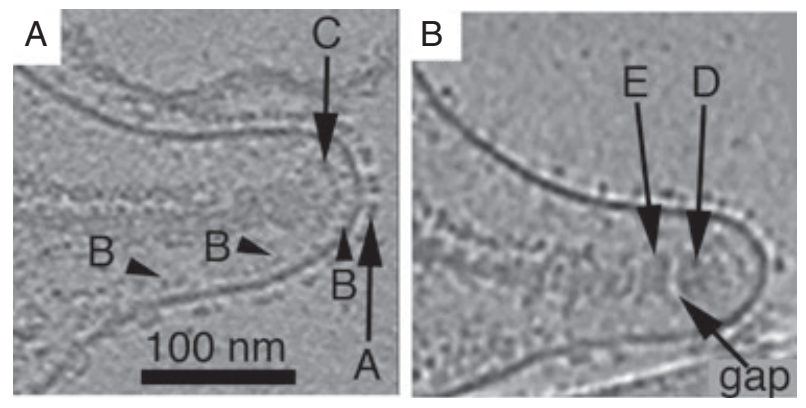

Fig. 4. Membrane proteins and terminal button. Slices through two different attachment organelles are shown and marked to highlight the membrane protein layers ' $A$ ' and ' $B$ ' and the three components of the terminal button ' $C$ '-' $E$ ' [the organelles in $(A)$ and $(B)$ are the same as those shown in Fig. $2 \mathrm{~A}$ and $\mathrm{E}$ respectively]. Capital letters: components as labelled in Fig. 20. 

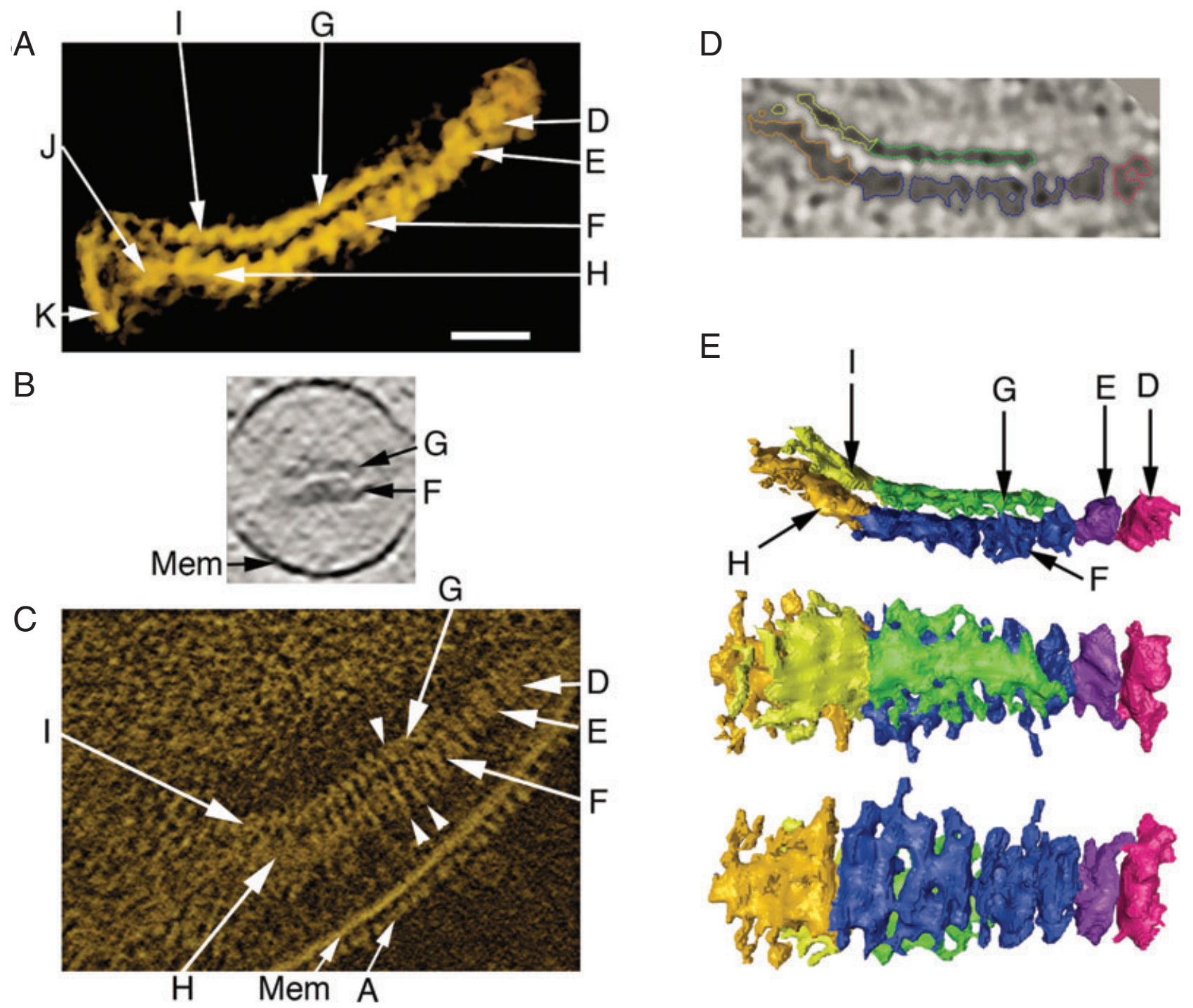

Fig. 5. Electron-dense core.

A. Volume-rendering of the electron-dense core and bowl complex shown in Fig. 2A, with components labelled.

B. Cross-section of the core shown in Fig. 2 I perpendicular to the long axis of the rods at a point distal to the bend. Mem, membrane.

C. A thin section through the attachment organelle shown in Fig. $2 \mathrm{~J}$, minimally denoised and volume-rendered to highlight the fine structure of the core's distinct segments (arrowheads).

D. Cross-section of the same attachment organelle after further denoising and automatic segmentation with a 'fill' tool to generate a surface. E. Surface-rendering, rotated and colour-coded as in Fig. 20 to give a sense of the gross structure from different views. Note that the structure will appear slightly elongated from the 'top' and 'bottom' views due to the missing wedge of data in electron tomography.

visible, large complexes such as ribosomes were clearly excluded from this region, which extended from component ' $\mathrm{C}$ ' to components ' $\mathrm{H}$ ' and ' $\mathrm{l}$ '. Again in contrast to an earlier report (Hegermann et al., 2002), no filamentous connections were found between the shaft of the electrondense core and the membrane.

\section{Replication of the attachment organelle}

Approximately half (five out of 12) of the observed cells had two electron-dense cores (see, for example, Fig. 7), and one cell had three. The cores were found separated by various distances, including at opposite ends of the cell (Fig. 1). No structural connections were seen between electron-dense cores within the same cell. In the cells where the attachment organelles protruded out away from the cell body and there were multiple cores, their proximal ends came nearest to each other, as this geometry requires. In contrast and not seen before, in the cells where their were multiple and internalized cores, the terminal buttons sometimes came nearest to each other. In the cell with three cores, two of the cores were close and 

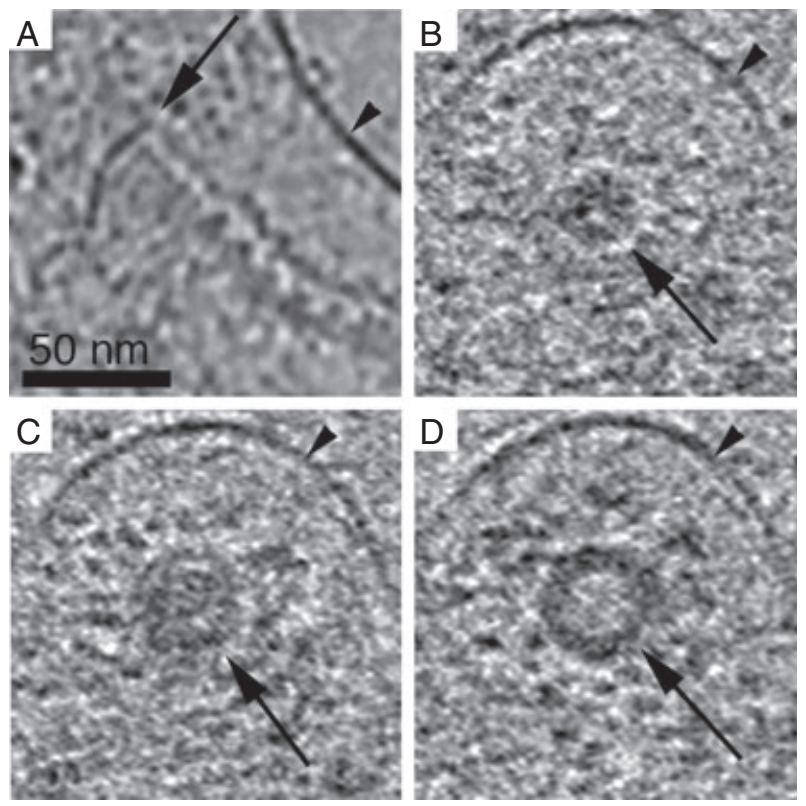

Fig. 6. Bowl complex.

A. 'Sagittal' section through the bowl complex and the proximal end of the electron-dense core shown in Fig. 2E.

B-D. 'Coronal' $1.6 \mathrm{~nm}$ sections (roughly perpendicular to the axis of the core) through the bowl complex at different positions, starting at the bottom of the bowl and moving up to its rim.

Arrows point to the bowl complex, while arrowheads point to the cell membrane.

formed a ' $V$ ' shape, abutting near the bowl complex and diverging towards the terminal button. The third core was more distant, and its terminal button pointed towards the distal ends of the other two. In the cells with multiple cores, bowl complexes were always seen with at least one but not necessarily all cores.

\section{Cytoskeleton filaments}

Previous studies on M. pneumoniae have often looked at the structure that remains after solubilizing the cell membrane with the detergent Triton X-100, and have repeatedly reported that $5 \mathrm{~nm}$ filaments were associated with the core (Meng and Pfister, 1980; Gobel et al., 1981; Hegermann et al., 2002). Loose bundles of $\sim 5 \mathrm{~nm}$ diameter filaments were also seen here in three cells (Fig. 8), but were not visibly connected to the core. Instead they were only seen where the cell body narrowed as it stretched across the supporting carbon film.

\section{Discussion}

Earlier preparative techniques of plastic-embedding and detergent removal of the membrane left the structural details of the attachment organelle uncertain because these methods disrupted native conditions and probably introduced artefacts. Here, cells were plunge-frozen and imaged in an intact, frozen-hydrated and therefore nearnative state. The cells we imaged were mostly unattached to any surface and had been dislodged from the culture flask before they were applied to the EM grid. This may explain why some of the cells were more pleomorphic than the rod-shapes seen before, and why some of the electron-dense cores were internalized rather than protruding from the main cell body. Nevertheless in all cases the electron-dense core was seen to be attached to the membrane by the terminal button, surrounded by an electron-lucent area, and accompanied by rows of surface proteins.

We found that the native attachment organelle is an enormous, complex, conformationally flexible molecular machine composed of at least 11 distinct regions. Previous observations of split electron-dense cores (Hegermann et al., 2002; Willby and Krause, 2002) led to the hypothesis that cores replicate through a semi-conservative mechanism, where the two rods of the core separate and each serves as a template to rebuild a partner (Krause and Balish, 2004). Our observations did not specifically support this model, because the two rods of the
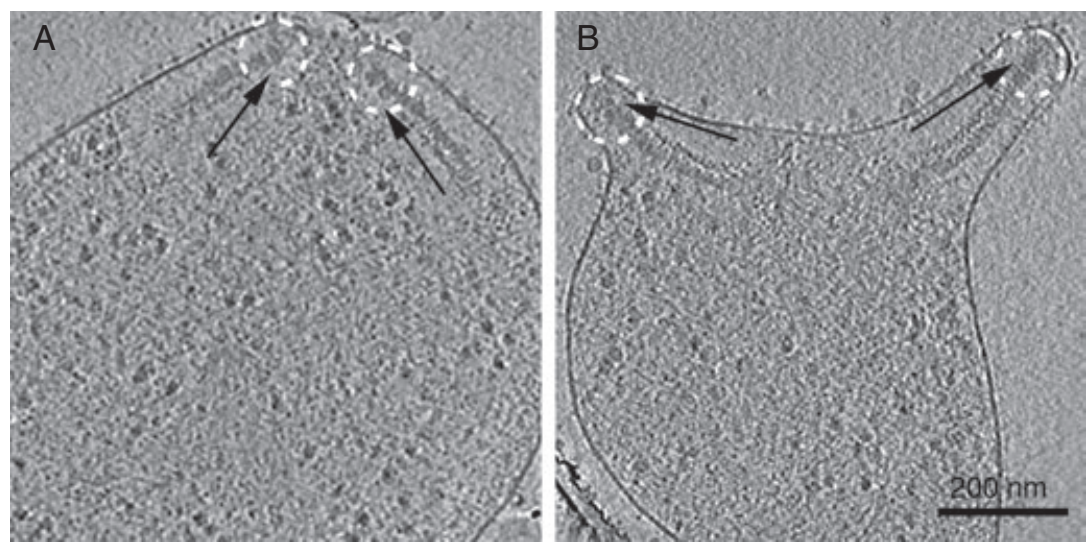

Fig. 7. Multiple electron-dense cores. Two cells with multiple electron-dense cores are shown where the terminal buttons (highlighted with white dotted circles) are oriented either towards (A) or away (B) from each other. The black arrows point from the bowl complex to the terminal button alongside the thin rods. The organelles in $(A)$ and $(B)$ are the same as those shown in Fig. $2 \mathrm{~L}$ and $\mathrm{M}$ and Fig. $2 \mathrm{~B}$ and $\mathrm{C}$ respectively. 

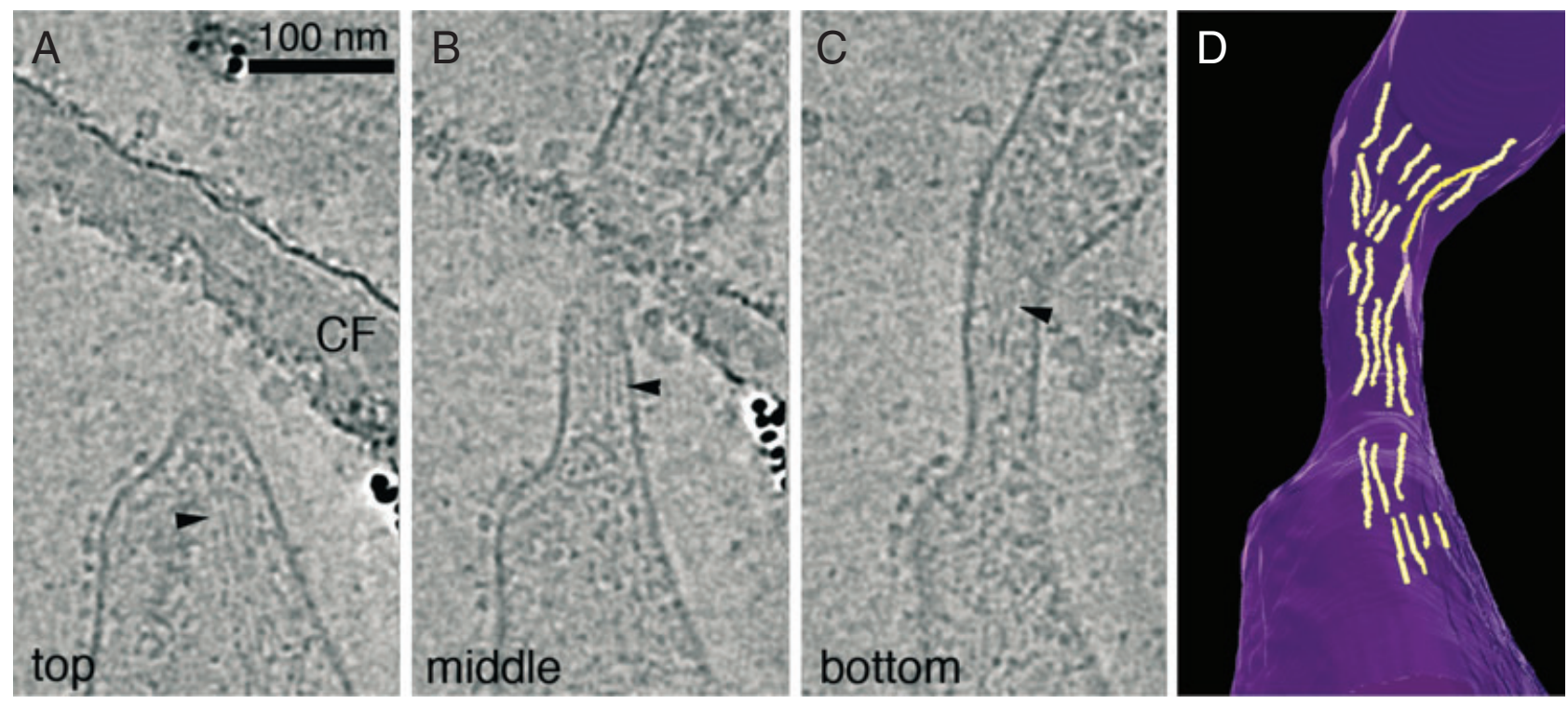

Fig. 8. Cytoskeleton filaments.

A-C. Serial sections through a tomogram of one cell showing a bundle of $5 \mathrm{~nm}$ filaments running through a point of cell narrowing. Arrowheads point to the fibre bundle. CF, carbon film.

D. Selective, manual, three-dimensional segmentation of some of the fibres (yellow). The membrane has been rendered purple.

core are not identical and no cores were observed with only one rod, even when the cores were in close proximity and had presumably just replicated.

While some information is available about the localization of proteins P1, B, C, P65, P30 and HMW1-3, the structural clues gathered here were insufficient to assign them to specific components. We can conclude, however, that the surface proteins are densely packed and must work as complexes, because the densities seen were too large to be individual proteins. The identity of the cytoskeletal filaments is particularly interesting. Of the known bacterial cytoskeletal proteins, only FtsZ (MPN 317) has been recognized in $M$. pneumoniae. Other potential candidates include EF-Tu (MPN 665), which has been shown to form filaments in vitro (Beck et al., 1978); and DnaK (MPN 434, also known as Hsp70), which has been characterized as a protein chaperone but is structurally homologous to actin (Flaherty et al., 1991). Interestingly, DnaK was also shown to be associated with $\mathrm{P} 1$ by chemical cross-linking (Layh-Schmitt et al., 2000). As each of these three candidate filament-forming proteins is near ubiquitous in prokaryotes, knowledge of their potentially filamentous nature and arrangement in vivo here could have widespread implications.

Why would one of the smallest and simplest of all cells construct an organelle with such a fantastic size and complexity? While the attachment organelle is known to be required for adhesion (Morrison-Plummer et al., 1986), this may only require localization of key surface proteins, and would by itself hardly require such a sophisticated structure. We considered the possibility that the two-rod core could be like a harpoon or crossbow, where one rod advanced with respect to the other to press against or puncture host cells. Nothing like this has ever been seen, however, in thin-section EM images of M.pneumoniae attached to tracheal epithelium (Wilson and Collier, 1976). Metabolic functions such as substrate channelling or histone-like DNA-organizing functions also seem unlikely.

Building on (i) published evidence suggesting that the attachment organelle is where the motive force in these cells is generated (Hasselbring et al., 2005; Seto et al., 2005a), (ii) mutational data showing that the core itself is required for motility (Balish et al., 2003; Balish and Krause, 2005), and (iii) our observations here of the complexity and conformational flexibility of the core, we propose that the core itself is the molecular motor that produces movement. We suggest a model in which the electron-dense core undergoes inchworm-like conformational changes that push the tip of the cell forward in small steps. Starting with surface proteins at the tip bound to a substrate and the core fully extended, the core may cyclically contract by bending at its various joints and/or minimizing the gaps between its segments, and then spring back to full length. When it springs back to full length, the bowl complex may provide leverage and resistance, like a paddle against water, especially if in fact the cytoplasmic filaments do indeed attach to it as suggested earlier (but not seen here, although if filaments bent rapidly they could have escaped our detection) and further gel the adjacent cytoplasm. Extension of the core would then require new membrane to 'roll' down from above in front of the terminal button, attracting a new plaque of surface adhesion pro- 

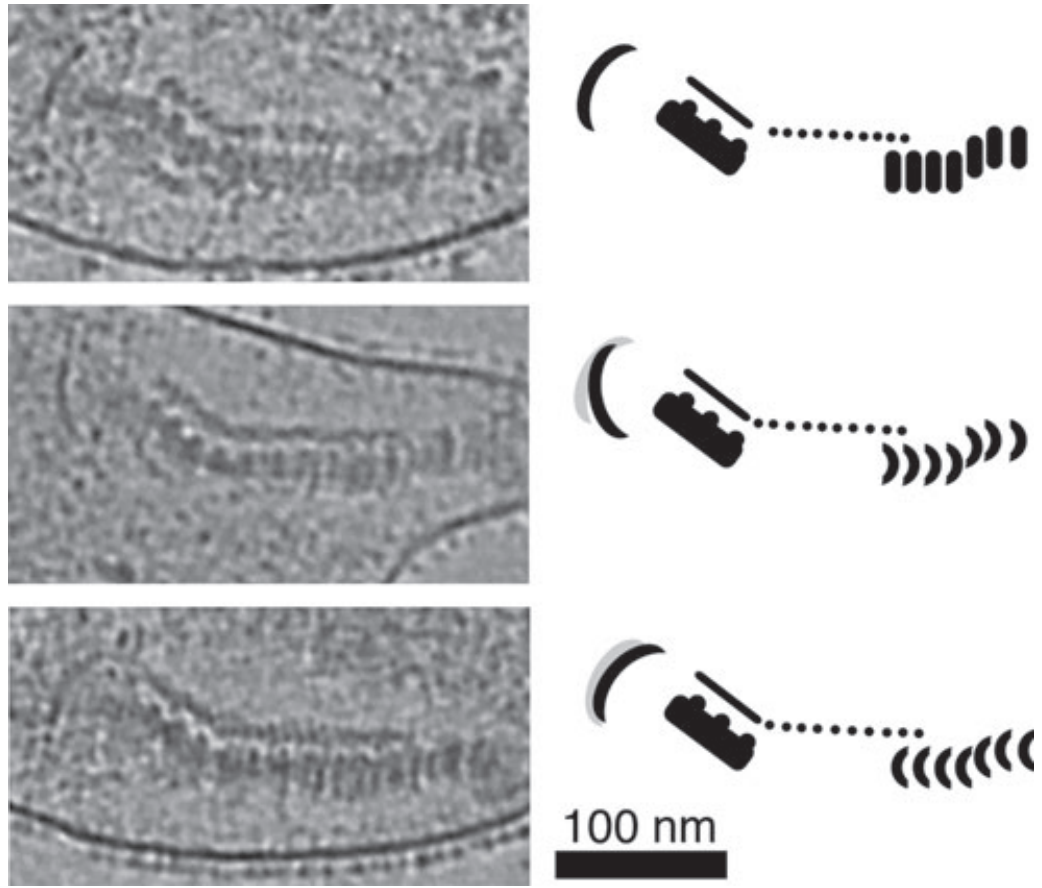

$100 \mathrm{~nm}$
Fig. 9. Evidence of conformational changes. The attachment organelles from Fig. 2K, F and $J$ are shown to the left of corresponding schematics highlighting conformational differences. The relative position of the first bowl complex is overlaid on the later two schematics as a grey shadow. For clarity the bowl complex is shown moving relative to a stable core, though the opposite, a stable bowl complex and mobile core, may be more likely. teins that might prevent regression. As the cell advanced and earlier contacts moved towards the rear, they might weaken and release, perhaps through loss of the organization originally imposed on them by other elements of the attachment organelle like the layer of submembrane proteins seen here (component 'B').

One attractive aspect of this model is that it could explain the otherwise mysterious electron-lucent area. Patterned beating of the core could clear the area of large macromolecules leaving only smaller molecules and water, just as any shaking tends to separate objects with different properties. This would be true regardless of whether the core was internalized within the cell body or protruding in a finger-like extension, just as we saw here. Published pictures of Mycoplasma penetrans, however, argue against this explanation. $M$. penetrans is a relative of $M$. pneumoniae, which also apparently excludes large macromolecules from its tip, but it lacks an analogous core. More specifically and in contrast to our results here, M. penetrans' tip has been described as filled with densely packed fine granules (Lo et al., 1992; Neyrolles et al., 1998). Until this structure is also imaged in its native state by ECT, conclusive comparisons are probably premature. In the absence of any membrane or protein boundary, an alternative explanation is that some sort of gel could actually be responsible for excluding large macromolecules in both species.

The core motility model also offers an explanation for the size and complexity of the core, the solid bowl complex and the organized rows of proteins both inside and outside the membrane. The motility of the attachment organelle might be important for cell division. The attachment organelle has been seen to replicate, migrate to opposite sides of predivisional cells, and then stay at the forefront of the daughter cells as they separate (Seto et al., 2001). It may actually pull the daughter cells apart. The bacterial genome may also attach to the organelle, perhaps via the bowl complex, to ensure chromosome segregation (Seto et al., 2001).

In an effort to identify conformational flexibility within the attachment organelle in support of our model, we found three types of evidence: (i) the spacing between the segments of the electron-dense core and also the nodules of the terminal button were variable, like an accordion; (ii) sometimes all the segments were straight, sometimes they were curved inwards, and sometimes they were curved outwards; and (iii) the position and orientation of the bowl complex varied (Fig. 9). Because the cells were unattached to any surface when imaged, however, these conformational changes may not be associated with those that occur during gliding motility. If our model is correct, we would also have expected larger variations as well. Perhaps they exist, but are so short-lived that none were captured and imaged here.

Other models of motility were considered. Current models for the motility of $M$. mobile propose that individual surface proteins cyclically stroke a surface, propelling the cell like the feet of a centipede (Uenoyama et al., 2004). While these proteins are localized to an elongated extension of the cell similar to the attachment organelle of M. pneumoniae they are excluded from its tip (Uenoyama and Miyata, 2005). No homologues to these proteins have 
been found in M.pneumoniae (Miyata, 2005) and M. mobile does not appear to have either an electrondense core or an electron-lucent area (Shimizu and Miyata, 2002). If the mechanisms in the two organisms were nevertheless similar, one wonders what would justify the size and complexity of the electron-dense core and explain the electron-lucent area. A conveyor-belt model for track-based motility (McBride, 2001) also seems discordant with our observations here because no array of structural links were seen between the shaft of the core and the rows of membrane proteins.

Our proposed model makes testable predictions. First, it predicts that the gliding motility in M. pneumoniae should be incremental with at least a roughly characteristic step size. Second, unlike strictly structural proteins, some component of the core must consume energy. Third, directed movement would require that the core contact a surface through adhesion proteins in a surrounding membrane (i.e. isolated cores might 'twitch', but not move forward steadily, and isolated membranes with their surface proteins would be motionless). Fourth, in contrast to individual surface proteins of current $M$. mobile models which would remain fixed relative to the tip, individual adhesion proteins labelled here would cycle from the tip of the attachment organelle towards the rear, then release the surface and diffuse back up to the front. More work is needed to identify the proteins that form each component and to test these predictions.

\section{Experimental procedures}

\section{Cultivation conditions}

Wild-type M. pneumoniae, cell strain M129 (Lipman et al., 1969), was cultured for $2-3$ days in $10 \mathrm{ml}$ of SP-4 medium at $37^{\circ} \mathrm{C}$ in a plastic culture flask $\left(25 \mathrm{~cm}^{2}\right)$ (Tully et al., 1977). Cells were then scraped off the culture flask and concentrated by centrifugation (10 $000 \mathrm{~g}$ for $3 \mathrm{~min}$ ).

\section{Electron cryotomography}

Concentrated M.pneumoniae were applied to glow-discharged Quantifoil (SPI Supplies) or lacy carbon (Ted Pella) grids previously treated with $10 \mathrm{~nm}$ gold fiducial markers. Excess liquid was removed and the samples were plungefrozen in liquid ethane using a Vitrobot (FEI). Maintaining the samples at liquid nitrogen temperature throughout the experiment, the grids were loaded into 'flip-flop' tilt rotation holders and loaded into a $300 \mathrm{kV}$, FEG, G2 Polara transmission EM (FEI). Image series were acquired at half to three degree intervals, tilting the sample between roughly $-62^{\circ}$ to $+62^{\circ}$, using the predictive UCSF tomography software package (Zheng et al., 2004). All images were zero-loss filtered with a slit-width of $20 \mathrm{eV}$. For some cells the grid was rotated $90^{\circ}$ between a first and second tilt-series (lancu et al., 2005). Images were acquired under low-dose conditions 10-30 $\mu \mathrm{m}$ underfocus and with a magnification such that after the energy filter, each pixel on the CCD represented between 0.56 and $0.82 \mathrm{~nm}$ on the specimen plane.

\section{Image analysis}

Images were aligned using gold fiducial markers. Single-axis tilt-series were reconstructed by weighted back-projection and dual-axis tilt-series were merged using IMOD (Mastronarde, 1997). Reconstructions were denoised using nonlinear anisotropic diffusion (Frangakis and Hegerl, 2001), distributed across the network of lab workstations with the Peach system (Leong et al., 2005). Images were produced using IMOD or the Amira software package (Mercury Computer Systems). All the images shown were denoised except for those in Fig. 1.

\section{Acknowledgements}

This work was supported in part by NIH Grant P01 G66521 to G.J.J., DOE Grant DE-FG02-04ER63785 to G.J.J., a Searle Scholar Award to G.J.J., and gifts to Caltech from the Ralph M. Parsons Foundation, the Agouron Institute, and the Gordon and Betty Moore Foundation. We thank Duncan C. Krause of the University of Georgia for providing M. pneumoniae M129, for repeated discussions, for sharing unpublished data, and for his reading of the manuscript.

\section{References}

Balish, M.F., and Krause, D.C. (2005) Mycoplasma attachment organelle and cell division. In Mycoplasmas: Molecular Biology Pathogenicity and Strategies for Control. Blanchard, A., and Browning, G. (eds). Wymondham: Horizon Bioscience, pp. 189-237.

Balish, M.F., Santurri, R.T., Ricci, A.M., Lee, K.K., and Krause, D.C. (2003) Localization of Mycoplasma pneumoniae cytadherence-associated protein HMW2 by fusion with green fluorescent protein: implications for attachment organelle structure. Mol Microbiol 47: 49-60.

Baseman, J.B., Cole, R.M., Krause, D.C., and Leith, D.K. (1982) Molecular basis for cytadsorption of Mycoplasma pneumoniae. J Bacteriol 151: 1514-1552.

Beck, B.D., Arscott, P.G., and Jacobson, A. (1978) Novel properties of bacterial elongation factor Tu. Proc Natl Acad Sci USA 75: 1250-1254.

Biberfeld, G., and Biberfeld, P. (1970) Ultrastructural features of Mycoplasma pneumoniae. J Bacteriol 102: 855-861.

Flaherty, K.M., McKay, D.B., Kabsch, W., and Holmes, K.C. (1991) Similarity of the three-dimensional structures of actin and the ATPase fragment of a $70-\mathrm{kDa}$ heat shock cognate protein. Proc Natl Acad Sci USA 88: 5041-5045.

Frangakis, A.S., and Hegerl, R. (2001) Noise reduction in electron tomographic reconstructions using nonlinear anisotropic diffusion. J Struct Biol 135: 239-250.

Fraser, C.M., Gocayne, J.D., White, O., Adams, M.D., Clayton, R.A., Fleischmann, R.D., et al. (1995) The minimal gene complement of Mycoplasma genitalium. Science 270: 397-404. 
Gobel, U., Speth, V., and Bredt, W. (1981) Filamentous structures in adherent Mycoplasma pneumoniae cells treated with nonionic detergents. J Cell Biol 91: 537-543.

Hasselbring, B.M., Jordan, J.L., and Krause, D.C. (2005) Mutant analysis reveals a specific requirement for protein P30 in Mycoplasma pneumoniae gliding motility. J Bacteriol 187: 6281-6289.

Hegermann, J., Herrmann, R., and Mayer, F. (2002) Cytoskeletal elements in the bacterium Mycoplasma pneumoniae. Naturwissenschaften 89: 453-458.

Himmelreich, R., Hilbert, H., Plagens, H., Pirkl, E., Li, B.C., and Herrmann, R. (1996) Complete sequence analysis of the genome of the bacterium Mycoplasma pneumoniae. Nucleic Acids Res 24: 4420.

lancu, C.V., Wright, E.R., Benjamin, J., Tivol, W.F., Dias, D.P., Murphy, G.E., et al. (2005) A 'flip-flop' rotation stage for routine dual-axis electron cryotomography. J Struct Biol 151: 288.

Krause, D.C., and Balish, M.F. (2004) Cellular engineering in a minimal microbe: structure and assembly of the terminal organelle of Mycoplasma pneumoniae. Mol Microbiol 51: 917-924.

Layh-Schmitt, G., Podtelejnikov, A., and Mann, M. (2000) Proteins complexed to the P1 adhesin of Mycoplasma pneumoniae. Microbiology 146: 741-747.

Leong, P.A., Heymann, J.B., and Jensen, G.J. (2005) Peach: a simple Perl-based system for distributed computation and its application to cryo-EM data processing. Structure 13: 505.

Lipman, R.P., Clyde, W.A., Jr and Denny, F.W. (1969) Characteristics of virulent, attenuated, and avirulent $\mathrm{Myco}-$ plasma pneumoniae strains. J Bacteriol 100: 1037-1043.

Lo, S.-C., Hayes, M.M., Tully, J.G., Wang, R.Y.-H., Kotani, H., Pierce, P.F., et al. (1992) Myoplasma penetrans sp. nov., from the urogenital tract of patients with AIDS. Int $J$ Syst Bacteriol 42: 357-364.

Lucic, V., Forster, F., and Baumeister, W. (2005) Structural studies by electron tomography: from cells to molecules. Annu Rev Biochem 74: 833-865.

McBride, M.J. (2001) Bacterial gliding motility: multiple mechanisms for cell movement over surfaces. Annu Rev Micobiol 55: 49-75.

Mastronarde, D.N. (1997) Dual-axis tomography: an approach with alignment methods that preserve resolution. J Struct Biol 120: 343.

Mattick, J.S. (2002) Type IV pili and twitching motility. Annu Rev Micobiol 56: 289-314.

Meng, K.E., and Pfister, R.M. (1980) Intracellular structures of Mycoplasma pneumoniae revealed after membrane removal. J Bacteriol 144: 390-399.

Miyata, M. (2005) Gliding motility of mycoplasmas: the mechanism cannot be explained by current biology. In Myco- plasmas: Molecular Biology Pathogenicity and Strategies for Control. Blanchard, A. and Browning, G., (eds). Wymondham: Horizon Bioscience, pp. 137-163.

Morrison-Plummer, J., Leith, D.K., and Baseman, J.B. (1986) Biological effects of anti-lipid and anti-protein monoclonal antibodies on Mycoplasma pneumoniae. Infect Immun 53: 398-403.

Neyrolles, O., Brenner, C., Prevost, M.-C., Fontaine, T., Montagnier, L., and Blanchard, A. (1998) Identification of two glycosylated components of Mycoplasma penetrans: a surface-exposed capsular polysaccharide and a glycolipid fraction. Microbiology 144: 1247-1255.

Seto, S., Layh-Schmitt, G., Kenri, T., and Miyata, M. (2001) Visualization of the attachment organelle and cytadherence proteins of Mycoplasma pneumoniae by immunofluorescence microscopy. J Bacteriol 183: 1621-1630.

Seto, S., Kenri, T., Tomiyama, T., and Miyata, M. (2005a) Involvement of P1 adhesin in gliding motility of Mycoplasma pneumoniae as revealed by the inhibitory effects of antibody under optimized gliding conditions. J Bacteriol 187: 1875-1877.

Seto, S., Uenoyama, A., and Miyata, M. (2005b) Identification of a 521-kilodalton protein (Gli521) involved in force generation or force transmission for Mycoplasma mobile gliding. J Bacteriol 187: 3502-3510.

Shimizu, T., and Miyata, M. (2002) Electron microscopic studies of three gliding mycoplasmas, Mycoplasma mobile, M. pneumoniae, and M. gallisepticum, by using the freeze-substitution technique. Curr Microbiol 44: 431434.

Tully, J., Whitcomb, R., Clark, H., and Williamson, D. (1977) Pathogenic mycoplasma: cultivation and vertebrate pathogenicity of a new spiroplasma. Science 195: 892-894.

Uenoyama, A., and Miyata, M. (2005) Identification of a 123kilodalton protein (Gli123) involved in machinery for gliding motility of Mycoplasma mobile. J Bacteriol 187: 55785584.

Uenoyama, A., Kusumoto, A., and Miyata, M. (2004) Identification of a 349-kilodalton protein (Gli349) responsible for cytadherence and glass binding during gliding of Mycoplasma mobile. J Bacteriol 186: 1537-1545.

Willby, M.J., and Krause, D.C. (2002) Characterization of a Mycoplasma pneumoniae hmw3 mutant: implications for attachment organelle assembly. J Bacteriol 184: 30613068.

Wilson, M.H., and Collier, A.M. (1976) Ultrastructural study of Mycoplasma pneumoniae in organ culture. J Bacteriol 125: 332-339.

Zheng, Q.S., Braunfeld, M.B., Sedat, J.W., and Agard, D.A. (2004) An improved strategy for automated electron microscopic tomography. J Struct Biol 147: 91-101. 